\title{
AN ANALYTICAL BENCHMARK FOR THE CALCULATION OF CURRENT DISTRIBUTION IN SUPERCONDUCTING CABLES
}

\author{
L. Bottura ${ }^{1}$, M. Breschi ${ }^{2}$, M. Fabbri ${ }^{2}$
}

The validation of numerical codes for the calculation of current distribution and AC loss in superconducting cables versus experimental results is essential, but could be affected by approximations in the electromagnetic model or incertitude in the evaluation of the model parameters. A preliminary validation of the codes by means of a comparison with analytical results can therefore be very useful, in order to distinguish among different error sources. We provide here a benchmark analytical solution for current distribution that applies to the case of a cable described using a distributed parameters electrical circuit model. The analytical solution of current distribution is valid for cables made of a generic number of strands, subjected to well defined symmetry and uniformity conditions in the electrical parameters. The closed form solution for the general case is rather complex to implement, and in this paper we give the analytical solutions for different simplified situations. In particular we examine the influence of different boundary conditions, the effect of a localised resistance in the middle of the cable such as in the case of quench and the effects of localized time dependent magnetic fluxes acting on the cable.

1 LHC Division, CERN, Geneva, Switzerland

2 DIE, Department of Electrical Engineering, University of Bologna, Italy

Presented at the 5th Workshop on Computation of Thermo-Hydraulic Transients in Superconductors (CHATS 2002)

16-18 September 2002, Karlsruhe, Germany

Administrative Secretariat

LHC Division

CERN

CH - 1211 Geneva 23
Geneva, Switzerland

20 December 2002 


\title{
An Analytical Benchmark for the Calculation of Current Distribution in Superconducting Cables
}

\author{
L. Bottura $^{\text {a }}$, M. Breschi ${ }^{\text {b,* }}$, M. Fabbri ${ }^{\text {b }}$ \\ ${ }^{a}$ CERN, LHC Division, Geneva, Switzerland \\ ${ }^{\mathrm{b}}$ DIE, Department of Electrical Engineering, University of Bologna, Italy \\ Prepared for publication in: \\ Cryogenics
}

\begin{abstract}
The validation of numerical codes for the calculation of current distribution and AC loss in superconducting cables versus experimental results is essential, but could be affected by approximations in the electromagnetic model or incertitude in the evaluation of the model parameters. A preliminary validation of the codes by means of a comparison with analytical results can therefore be very useful, in order to distinguish among different error sources. We provide here a benchmark analytical solution for current distribution that applies to the case of a cable described using a distributed parameters electrical circuit model. The analytical solution of current distribution is valid for cables made of a generic number of strands, subjected to well defined symmetry and uniformity conditions in the electrical parameters. The closed form solution for the general case is rather complex to implement, and in this paper we give the analytical solutions for different simplified situations. In particular we examine the influence of different boundary conditions, the effect of a localised resistance in the middle of the cable such as in the case of quench and the effects of localized time dependent magnetic fluxes acting on the cable.
\end{abstract}

Keywords: Superconducting cables (A), Electromagnetic phenomena (C), Accelerator magnets (F), Fusion magnets (F) 


\section{Introduction}

Cables for high current applications consist invariably of many superconducting strands in the form of a cable bundle where the strands are connected in parallel. In order to reduce the induced currents and the corresponding losses when exposed to external field changes, the strands are twisted to achieve transposition. This fabrication technique results in a wide variety of cable configurations of which Rutherford cables for accelerator magnets and Cable-in-ConduitConductors (CICC) for fusion magnets are two typical examples. For any possible configuration the question on how the current distributes among the parallel strands may be of vital importance. Several experiments and theoretical works have shown that non uniform current distribution among the strands of Rutherford cables can lead to the development of a longitudinal oscillation of the field harmonics in the bore of accelerator dipoles, usually referred to as "magnetic field pattern" [1]. It has also been shown that the decay of the field harmonics measured during constant field phases such as the phase of injection of particle beams into the accelerator is related through strand magnetization to the current distribution among the cable strands [2,3]. These phenomena must be predicted and corrected for the safe operation of a particle accelerator.

On the fusion magnets side, it has been established experimentally that CICC conductors may show a degradation of their quench performance when current distribution and re-distribution is hindered by large interstrand resistance. The degradation appears in steady state as a modification of the current-voltage characteristics of the cable, that has lower $n$-value of the power law compared to single strands, and during fast transients, as a ramp-rate dependent quench current, significantly lower than the critical current of individual strands. Uneven current distribution is discussed as one of the possible mechanisms for this performance degradation. For this reason several dedicated experiments were designed and performed to analyse the relation between the conductor thermal stability and the current distribution in the cable. An extensive review of the experimental work can be found in [4].

In parallel, several research groups have developed numerical codes for the calculation of current distribution and ac-losses in multistrand cables. Some of these codes are based on lumped parameters circuit models of cables [5-9], while others rely on distributed parameters models [1012]. Despite this large experimental and theoretical effort, there is a lack of simple quantitative formulae for the estimation of current non uniformities in real-scale conductors. Moreover, little progress has been made to determine design criteria of practical use to improve the conductor design in order to limit the performance degradation due to uneven current distributions.

Some interesting hints in this direction can be given by analytical work. Analytical studies were limited up to now to simple cables made of a few strands, typically two, in which case the governing equations simplify to a great extent [13-16]. In order to extend the reach of analytical models we have developed an analytical solution for the partial differential equations describing current diffusion in the frame of a distributed parameters model [17]. This solution applies to cables made of a generic number of strands, and is valid when the cable inductance, resistance and conductance matrices satisfy specific symmetry and circularity conditions. The validity of these conditions in most practical cases is discussed in [17].

As the analytical solution is rather complex to implement in its general form, involving intricate mathematical functions and the numerical calculation of integrals, in this paper we provide simplified analytical formulae for the calculation of current distribution in three different relevant cases. The first case is representative of uneven boundary conditions imposed at the cable ends. The second case is representative of the initial phases of the quench of one strand. The third case is 
representative of a driving voltage acting on one strand in the presence of a time dependent magnetic field, due to a transposition error with respect to the field itself.

These formulae can be used to build an analytical benchmark for the validation of numerical codes modelling current distribution in multistrand cables. The equations used to describe analytically current diffusion are quite general, and this benchmark applies to most of the codes based on distributed parameters models developed in the past years. In addition the analytical solutions reported here give a basis for useful scalings for the behaviour of multistrand cables.

\section{Model and assumptions}

The model for the current distribution that we have developed $[12,18]$ is shown schematically in Fig. 1 (for three strands). The cable has a total length $L$ and is composed of $N$ strands. It is assumed that the current can flow continuously from each strand to all the other strands through distributed contacts. These contacts are taken into account through longitudinal conductances per unit length $g_{h, k}$ (with $h, k=1, N$ ) between the $h$-th and $k$-th strand. The local mutual inductive effect between strand currents is taken in account by means of a matrix of per unit length mutual induction coefficients $l_{h, k}$ (with $h, k=1, N$ ). The voltages induced by external sources, such as, e.g., a time dependent external magnetic field $d B / d t$, are also distributed along the cable length and they are taken in account by means of external voltages per unit length $v_{h}^{\text {ext }}$ (with $h=1, N$ ). The equations of current diffusion in the presence of a longitudinal resistance describing the non linear current-voltage characteristics of each strand can be easily derived [12]. In the scope of the present study, the longitudinal resistance is neglected, i.e. the strands are considered to be in the perfectly superconducting state. Under this hypothesis, the current in each strand can be described by the following parabolic system of partial differential equations:

$\mathbf{g l} \frac{\partial \mathbf{i}}{\partial t}+\frac{\partial^{2} \mathbf{i}}{\partial x^{2}}-\mathbf{g v}^{e x t}=0$

where $t$ is time, $x$ is the distance along the cable axis, $\mathbf{i}$ is the vector that contains the $N$ strand currents, while $\mathbf{g}$ and $\mathbf{l}$ are the cable conductance and inductance matrices.

$\mathbf{I}=\left\lfloor l_{h, k}\right\rfloor$ with $h, k=1, N$

$\mathbf{g}=\left[\begin{array}{llll}-\sum_{\substack{k=2 \\ k \neq 1}}^{N} g_{1, k} & g_{1,2} & \cdots & g_{1, N} \\ g_{2,1} & -\sum_{\substack{k=1 \\ k \neq 2}}^{N} g_{2, k} & \cdots & g_{2, N} \\ \vdots & & & \\ g_{N, 1} & g_{N, 2} & \cdots & -\sum_{\substack{k=1 \\ k \neq N}}^{N} g_{N, k}\end{array}\right]$

The $l_{h, k}$ in (2) are the inductance coefficients among strands, defined for a unit of strand length. In order to facilitate the analytical approach, we assume that the model matrices $\mathbf{g}$ and $\mathbf{I}$ have a very simple structure. In particular we assume the inductances matrix $\mathbf{I}$ to be made of only two possible values, namely a self and a mutual induction coefficient, or:

$l_{h, h}=l$ and $l_{h, k}=m$ with $h, k=1, N, h \neq k$. 
The contact conductances between all different strand pairs are assumed to be identical, so that matrix $\mathbf{g}$ can be written as follows:

$\mathbf{g}=\left[\begin{array}{lllll}-(N-1) g & g & \cdots & g \\ g & -(N-1) & g & \cdots & g \\ \vdots & & & & \\ g & g & \cdots & -(N-1) & g\end{array}\right]$

In order to find the analytical solution of system (1) it is necessary to set the initial current distribution among the cable strands. We assume the initial currents in the cable to be equal to zero:

$i_{h}(x, 0)=0$ with $h=1, N$

This is obviously true in a real cable before any current cycle, or after the decay of all the current loops induced during previous current cycles.

All the assumptions made above, i.e. nil strand longitudinal resistance and initial current distribution, and the simplified feature of the model matrices, are not necessary for the derivation of the analytical solution of the system equations [17]. In particular, both assumptions (4) and (5) are true in the case of simple cables such as triplets or quadruplets, but are not likely to be fulfilled in a multi-stage cable, where the contact conductances and mutual inductances are 'graded'.

These choices are however useful as they result in a very relevant simplification of the analytical formulae discussed here, without affecting the validity of the benchmark proposed. In fact, these simplifications can also be considered for the model matrices in the numerical simulations, allowing in any case meaningful comparisons with the analytical solutions.

Moreover, numerical simulations show that these formulae are representative of the average behaviour of the strand currents evolution in the different situations discussed. This result can be obtained through an appropriate choice of the averaged parameters for the matrices $\mathbf{l}$ and $\mathbf{g}$. These parameters can be obtained imposing the conservation of the energy accumulated and dissipated in the cable in the passage from the real to the simplified matrices.

\section{Non uniform current distribution at the cable boundaries}

In this section we give the analytical solution for the evolution of the strand currents in a $\mathrm{N}$ strand cable subjected to an uneven current distribution at the cable ends. In particular we suppose that at one cable end only one of the $N$ cable strands is connected to the current leads and thus carries the whole transport current. At the other end we assume that all the strands are connected to the current leads and share the transport current uniformly distributed. The external voltage excitation $\mathbf{v}^{e x t}$ is assumed to be zero, neglecting the effect of self and external fields. The boundary conditions of the problem are schematically shown in Fig. 2. The equations, initial conditions and boundary conditions considered for this case can be written in the following form:

$$
\left\{\begin{array}{l}
\mathbf{g} \mathbf{l} \frac{\partial \mathbf{i}}{\partial t}(x, t)+\frac{\partial^{2} \mathbf{i}}{\partial x^{2}}(x, t)=0 \\
\mathbf{i}(x, t=0)=0 \\
i_{1}(x=0, t)=i_{o p}(t), \quad i_{h}(x=0, t)=0 \quad h=2, N \\
i_{h}(x=L, t)=\frac{i_{o p}(t)}{N} \quad h=1, N
\end{array}\right.
$$

where $L$ is the cable length and $i_{o p}$ is the total cable operating current. The detailed derivation of the solution for any kind of current cycle that can be found elsewhere [19]. Here we give the solution for the case of a current cycle made of a current ramp up with ramp rate $\beta$ until $t=t_{1}$ followed by a constant current phase at $t>t_{1}$, or: 
$i_{o p}(t)=\beta t$ for $t \leq t_{1}$

$i_{o p}(t)=\beta t_{1}$ for $t>t_{1}$

The currents in the strands during the current ramp are given by:

$$
\begin{aligned}
& i_{1}(x, t)=i_{o p}(t)\left(1-\frac{N-1}{N} \frac{x}{L}\right)-\frac{N-1}{N} 2 \beta \sum_{n=1}^{\infty} \frac{\tau}{n^{3} \pi} \sin \left(\frac{n \pi x}{L}\right)\left[1-\exp \left(\frac{-t n^{2}}{\tau}\right)\right] \\
& i_{h}(x, t)=i_{o p}(t) \frac{x}{N L}+\frac{1}{N} 2 \beta \sum_{n=1}^{\infty} \frac{\tau}{n^{3} \pi} \sin \left(\frac{n \pi x}{L}\right)\left[1-\exp \left(\frac{-t n^{2}}{\tau}\right)\right] \quad h=2, N
\end{aligned}
$$

while during the current plateau the currents are given by:

$$
\begin{aligned}
& i_{1}(x, t)=i_{o p}(t)\left(1-\frac{N-1}{N} \frac{x}{L}\right)-\frac{N-1}{N} 2 \beta \sum_{n=1}^{\infty} \frac{\tau}{n^{3} \pi} \sin \left(\frac{n \pi x}{L}\right)\left[\exp \left(\frac{-\left(t-t_{1}\right) n^{2}}{\tau}\right)-\exp \left(\frac{-t n^{2}}{\tau}\right)\right] \\
& i_{h}(x, t)=i_{o p}(t) \frac{x}{N L}+\frac{1}{N} 2 \beta \sum_{n=1}^{\infty} \frac{\tau}{n^{3} \pi} \sin \left(\frac{n \pi x}{L}\right)\left[\exp \left(\frac{-\left(t-t_{1}\right) n^{2}}{\tau}\right)-\exp \left(\frac{-t n^{2}}{\tau}\right)\right] \quad h=2, N
\end{aligned}
$$

In equations (10) through (13) $\tau$ represents the time constant of the cable, given by:

$$
\tau=N(l-m) g\left(\frac{L}{\pi}\right)^{2}
$$

As discussed in [17], in the simple case considered (uniform inductance and conductance matrices) this is the only time constant of the system. From the above equations we remark that the deviation of the strand currents from uniform distribution has two components. The first one, the first term on the right hand side of Eqs. (10)-(13), is a linear function of space constrained by the two values at the boundaries. The second component is a deviation from linearity that becomes negligible at times much longer than the cable time constant. Considering the solution during the ramp, Eqs. (10) and (11), it can be shown that the series converges to the following polynomial in $x$ :

$\lim _{t \rightarrow \infty} \sum_{n=1}^{\infty} \frac{\tau}{n^{3} \pi} \sin \left(\frac{n \pi x}{L}\right)\left[1-\exp \left(\frac{-t n^{2}}{\tau}\right)\right]=\frac{\pi^{2}}{12} \tau \frac{x}{L}\left(1-\frac{x}{L}\right)\left(2-\frac{x}{L}\right)$

This value is constant in time, while the linear term containing $i_{o p}(t)$ always increases in time. Hence after a sufficiently long time the non linear term can be neglected with respect to the linear one. During the current plateau the exponential present in the series reported in (12) and (13) forces this term to zero at times much longer than $\tau$. The current distribution in the limiting case of a long current ramp or a long current plateau is therefore given by a simple linear space dependence in all strands.

In order to apply these analytical formulae to the benchmarking of a numerical code, we have performed a set of numerical simulations involving cables made of different number of strands. A cable of length $L=2.3 \mathrm{~m}$ is subjected to a current cycle characterized by the parameters $\beta$ equal to $600 \mathrm{~A} / \mathrm{s}$ and $t_{1}$ equal to $10 \mathrm{~s}$ with the boundary conditions previously described. The number of strands is taken as a parameter in the simulations, keeping the inductances to the values $l=0.5 \mu \mathrm{H}$ and $m=0.25 \mu \mathrm{H}$, and the contact conductance to the value $g=7.463 \mathrm{MS}$. This choice results in $\tau$ equal to $2 \mathrm{~s}$ for the two-strand cable. We show in Fig. 3 the space dependence of the current in strand \#1 of a two-strand cable for the ramp and plateau phases respectively. Each curve 
plotted in Fig. 3 is normalised to the instantaneous value of the current at the beginning of the cable $(x=0)$ at each time step considered. The current imbalance at the boundaries disappears as expected along the cable length. As already anticipated, the strand current tends towards a perfectly linear variation, both during the current ramp and during the current plateau. We finally plot in Fig. 4 the comparison between the analytical solution and the numerical simulation for cables made of 2, 3, 4 and 5 strands. The numerical code used for comparison is based on a finite element approach and is described in detail in [18]. A very good agreement is obtained between the analytical solution and the numerical simulation.

\section{Local quench in one strand}

For this case a lumped longitudinal resistance $R$ is introduced in strand $\# 1$ in the middle of the cable $(x=L / 2)$, as shown in Fig. 5. This resistance is representative of a normal zone that can develop in one strand due to a direct heat input or to a local increase of current above the current sharing threshold. In the present model, the resistance is kept constant in time, neglecting the longitudinal propagation of the normal zone. This situation is representative of the first phases of quench of one strand before the normal zone can spread across the cross section of the cable, or downstream due to the heat conduction or a heating induced flow of helium. Also in this case the external voltage excitation is assumed to be absent for simplicity. A uniform current distribution is taken at the cable boundaries. The procedure for the determination of the analytical solution in this case is more complicated than in the first case, requiring the intervention of Laplace transform and is reported elsewhere [19]. We only report here the strand currents arising in a current cycle made of a current ramp followed by a current plateau as already described in section 3 .

Once the non-dimensional parameter $\omega=-R g L(N-1) / 4$ is defined, the solution for the strand currents during the current ramp-up $\left(t \leq t_{1}\right)$ can be written in the following way:

$$
\begin{aligned}
& i_{1}(x, t)=\frac{i_{o p}(t)}{N}\left(1+\frac{2 x}{L} \frac{\omega}{1-\omega}\right)+\frac{\beta}{N} A(x, t, \omega) \\
& i_{h}(x, t)=\frac{i_{o p}(t)}{N}\left(1-\frac{1}{N-1} \frac{2 x}{L} \frac{\omega}{1-\omega}\right)-\frac{\beta}{N(N-1)} A(x, t, \omega) \quad h=2, N
\end{aligned}
$$

for $0 \leq x \leq L / 2$ and:

$$
\begin{aligned}
& i_{1}(x, t)=\frac{i_{o p}(t)}{N}\left(1+2 \frac{L-x}{L} \frac{\omega}{1-\omega}\right)+\frac{\beta}{N} A(L-x, t, \omega) \\
& i_{h}(x, t)=\frac{i_{o p}(t)}{N}\left(1-\frac{1}{N-1} 2 \frac{L-x}{L} \frac{\omega}{1-\omega}\right)-\frac{\beta}{N(N-1)} A(L-x, t, \omega) \quad h=2, N
\end{aligned}
$$

for $L / 2<x \leq L$.

During the current plateau $\left(t>t_{1}\right)$ the strand currents are expressed as follows:

$$
\begin{aligned}
& i_{1}(x, t)=\frac{i_{o p}(t)}{N}\left(1+\frac{2 x}{L} \frac{\omega}{1-\omega}\right)+\frac{\beta}{N}\left[A(x, t, \omega)-A\left(x, t-t_{1}, \omega\right)\right] \\
& i_{h}(x, t)=\frac{i_{o p}(t)}{N}\left(1-\frac{1}{N-1} \frac{2 x}{L} \frac{\omega}{1-\omega}\right)-\frac{\beta}{N(N-1)}\left[A(x, t, \omega)-A\left(x, t-t_{1}, \omega\right)\right] \quad h=2, N
\end{aligned}
$$


for $0 \leq x \leq L / 2$, and:

$$
\begin{aligned}
& i_{1}(x, t)=\frac{i_{o p}(t)}{N}\left(1+2 \frac{L-x}{L} \frac{\omega}{1-\omega}\right)+\frac{\beta}{N}\left[A(L-x, t, \omega)-A\left(L-x, t-t_{1}, \omega\right)\right] \\
& i_{h}(x, t)=\frac{i_{o p}(t)}{N}\left(1-\frac{1}{N-1} 2 \frac{L-x}{L} \frac{\omega}{1-\omega}\right)-\frac{\beta}{N(N-1)}\left[A(L-x, t, \omega)-A\left(L-x, t-t_{1}, \omega\right)\right] h=2, N
\end{aligned}
$$

for $L / 2<x \leq L$.

The function $A(x, t, \omega)$ in equations (16) through (19) is given by:

$$
A(x, t, \omega)=2 \sum_{n=1}^{\infty} \frac{\cos \left(\xi_{n}(\omega)\right) \sin \left(\xi_{n}(\omega) \cdot 2 x / L\right)}{\cos \left(\xi_{n}(\omega)\right) \sin \left(\xi_{n}(\omega)\right)-\xi_{n}(\omega)}(-\tau)\left(\frac{\pi / 2}{\xi_{n}(\omega)}\right)^{2}\left(\exp \left(-\frac{t}{\tau}\left(\frac{\xi_{n}(\omega)}{\pi / 2}\right)^{2}\right)-1\right)
$$

Finally, the set $\xi_{n}(\omega), n=1, \infty$ is the solution of the following transcendental equation:

$$
\cot x=\frac{\omega}{x}
$$

where $x$ is an arbitrary, real positive unknown. As shown in Fig. 6, the solutions of this equation, denoted by $\xi_{n}(\omega)$ for $n=1,2, \ldots$, are bounded by $\left(n-\frac{1}{2}\right) \pi<\xi_{n}(\omega)<n \pi$. For large values of $n$ the solution is given by the simple limit:

$$
\lim _{n \rightarrow \infty} \xi_{n}(\omega)=\left(n-\frac{1}{2}\right) \pi \text {. }
$$

It is worth noting that also in this case the deviation of the strand currents from a uniform distribution is given by two terms clearly readable in Eqs. (16) through (19). The first term represents a linear variation of the current along the cable length, while the second term represents a deviation from linearity that becomes negligible with respect to the first term for times much longer than the cable time constant, both during the current ramp and during the current plateau. Arguments similar to those discussed in the previous section apply to the series in Eqs. (16) through (19), that can be shown to be formally identical to the series in Eq. (10).

We have applied the solution above to the analysis of current distribution considering the same ramp cycle and the same cable analysed in Section 3. A lumped resistance $R$ of $5 \mu \Omega$ is placed in strand \#1 in the middle of the cable. This situation corresponds to a normal zone with distributed resistance of $0.5 \mathrm{~m} \Omega / \mathrm{m}$ spreading over a length of $1 \mathrm{~cm}$ in strand $\# 1$, that is introduced in the simulations performed with the numerical code. The current in strand \#1 during the current rise and plateau is represented in Fig. 7. The current drops close to the lumped resistance diffusing from strand $\# 1$ to the other strands along a characteristic redistribution length that depends on the contact conductance among the strands. A comparison between the analytical solution and numerical simulation is shown in Fig. 8 for cables made of 2, 3, 4, and 5 strands with the same contact conductance and cable inductances as considered for the two-strand cable. A good agreement is found also in this case between the two solutions. 


\section{External voltage excitation}

We finally consider the cable subjected to a longitudinal voltage excitation localised over a short length $\delta$ placed in the middle of the cable and acting for a time $t_{1}$. This situation is representative of the voltage induced by a changing external magnetic flux in the presence of a localised transposition error in the cable. In particular we consider that the external voltage source acts only on one strand, e.g., the first one in the cable. A uniform current distribution is assumed at the cable boundaries.

The problem can then be stated as follows:

$$
\left\{\begin{array}{l}
\mathbf{g} \mathbf{l} \frac{\partial \mathbf{i}}{\partial t}(x, t)+\frac{\partial^{2} \mathbf{i}}{\partial x^{2}}(x, t)-\mathbf{g v}^{e x t}(x, t)=0 \\
\mathbf{i}(x, t=0)=0 \\
i_{h}(x=0, t)=i_{h}(x=L, t)=\frac{i_{o p}(t)}{N} \quad \text { with } h=1, N \\
v_{1}^{\text {ext }}=\frac{\mathrm{V}_{1}^{\text {ext }}}{\delta} \text { for } \quad x \in\left[\frac{L-\delta}{2}, \frac{L+\delta}{2}\right], v_{1}^{\text {ext }}=0 \quad \text { for } x \in\left[0, \frac{L-\delta}{2}\right] \text { and } x \in\left[\frac{L+\delta}{2}, L\right] \\
v_{h}^{\text {ext }}=0 \quad \text { for } x \in[0, L] \quad \text { with } h=2, N
\end{array}\right.
$$

The solution for this case is given by the following equations, for $t \leq t_{1}$ :

$$
i_{h}(x, t)=\frac{4}{\pi \alpha} I_{h} \sum_{\substack{n=1 \\ n \text { odd }}}^{\infty}\left[\frac{1}{n^{2}}\left(1-e^{-\frac{t}{\tau_{n}}}\right) \sin \left(\frac{n \alpha x}{w}\right) \sin (n \alpha)\right] \quad \text { with } h=1, N
$$

and for $t>t_{1}$ :

$$
i_{h}(x, t)=\frac{4}{\pi \alpha} I_{h} \sum_{\substack{n=1 \\ n \text { odd }}}^{\infty}\left[\frac{1}{n^{2}}\left(1-e^{-\frac{t_{1}}{\tau_{n}}}\right) e^{-\frac{\left(t-t_{1}\right)}{\tau_{n}}} \sin \left(\frac{n \alpha x}{w}\right) \sin (n \alpha)\right] \quad \text { with } h=1, N
$$

where we have used the following definitions for the parameters appearing in (24) and (25):

$$
\begin{aligned}
\alpha & =\pi \frac{L-\delta}{2 L} \\
w & =\frac{L-\delta}{2} \\
\tau_{n} & =\frac{\tau}{n^{2}}
\end{aligned}
$$

and $\tau$ is defined in (14).

The currents $I_{h}$ in (24) and (25) are the regime currents that are reached after a time much longer than the time constant $\tau$ and can be written as follows for the first strand:

$I_{1}=(N-1) \frac{w g V^{e x t}}{2}$. 
In all the other strands the regime current is the same and is given by:

$$
I_{h}=-\frac{w g V^{e x t}}{2} \quad \text { with } h=2, N
$$

As in the previous sections, we have applied these solutions to the benchmarking of the numerical code. The current distribution is calculated in the reference cable previously described with different number of strands (ranging from 2 to 5). A voltage source of $10 \mu \mathrm{V} / \mathrm{m}$ is localised in the center of the cable along a length $\delta=0.1 \mathrm{~m}$ and acts for a time $t_{1}$ equal to $10 \mathrm{~s}$. We show in Fig. 9 the evolution of the current in strand \#1 calculated with the two-strand cable. The current rises under the effect of the external voltage and then drops to zero after the end of the field ramp. In the simple case considered, with uniform cable inductance and conductance matrices, the currents in all strands except for strand $\# 1$ are coincident. The current in strand $\# 1$ increases until $t_{1}$ due to the external voltage excitation and then decays after $t_{1}$ with the time constant $\tau$. The current in all the other strands is negative and the absolute value is simply given by the current in the first strand divided by $(N-1)$, so that the total cable current is zero. A comparison between the analytical solution and the numerical simulation is shown in Fig. 10. A very good agreement is found also in this case between the analytical and numerical solution.

\section{Conclusions}

A set of analytical solutions for the equations of current diffusion in multistrand superconducting cables has been reported. These formulae extend previous analytical work performed for two-strand cables to cables made of a generic number of strands under some simplifying assumptions over inductance and conductance matrices. These solutions therefore provide an ideal analytical benchmark that can be used to test numerical codes for the calculation of current distribution in superconducting cables, in order to obtain a preliminary validation of these codes. The formulae are not intended to substitute numerical codes as they are not able to deal with the intrinsic non linearity of the current-voltage characteristics of superconducting strands, that becomes important as the strand current approaches its critical value. However, they can be useful for a rapid evaluation of the time scales and characteristic lengths of current redistribution for strands in superconducting regime, or when only a small portion of a strand turns normal due to an external disturbance. A possible future development of this work is the definition of improved design criteria for superconducting cables taking into account the effect of uneven current distributions between strands.

\section{References}

[1] A. K. Ghosh, K. E. Robins and W. B. Sampson. Axial variations in the magnetic field of superconducting dipoles. Supercollider, 1992; 4: 765.

[2] R. Wolf. The Decay of the Field Integral in Superconducting Accelerator Magnets wound with Rutherford Cables. Proc. MT15, 1997, Beijing, China.

[3] M. Haverkamp, A. Kuijper, A. Den Ouden, B. ten Haken, L. Bottura and H. H. J. ten Kate. Interaction between Current Imbalance and Magnetization in LHC cables. IEEE Trans. Appl. Sup., 2001; 11: 1609.

[4] N. Amemiya. Overview of current distribution and re-distribution in superconducting cables and their influence on stability. Cryogenics, 1998; 38: 545.

[5] A. Akhmetov. Network models of superconducting cables and the results of the matrix approach to their description. Physica C, 1998; 310: 309.

[6] F. Bellina, D. Boso, B.A. Schrefler, G. Zavarise. Modelling a Multistrand SC Cable with an Electrical Lumped Network. IEEE Trans. Appl. Sup., 2002; 12 (1): 1408. 
[7] A. Nijhuis, H. G. Knoopers, B. ten Haken, H. H. J. ten Kate. Model Study on AC Loss and Current Distribution ina Superconducting MultiStrand Cable. Report UT-NET 2000-1. 2000; Faculty of Applied Physics, University of Twente, Netherlands.

[8] D. Ciazynski, J. L. Duchateau. Validation of the CEA Electrical Network Model for the ITER Coils. IEEE Trans. Appl. Sup., 2001; 11: 1530.

[9] C. Meinecke, A. M. Miri, R. Petranovic. Numerical Investigation of the Current Distribution in Cable-inConduit-Conductors using Lumped Network Models. IEEE Trans. Appl. Sup., 2001; 11: 2579.

[10] N. Mitchell. Modelling of non-uniform current diffusion coupled with thermohydraulic effects in superconducting cables. Cryogenics. 2000; 40: 637.

[11] P. L. Ribani. CDCABLE: A code to calculate current distribution in superconducting multifilamentary cables. EFDA Task N. TWO - T400 - 1/01. 2002; Department of Electrical Engineering, University of Bologna, Italy.

[12] A. Akhmetov, L. Bottura, M. Breschi. A Continuum Model for Current Distribution in Rutherford Cables. IEEE Trans. Appl. Sup., 2001; 11: 2138.

[13] B. Turck. Influence of a transverse conductance on current sharing in a two-layer superconducting cable. Cryogenics. 1974; 24: 448.

[14] G. Ries. Stability in superconducting multistrand cables. Cryogenics, 1980; 20: 513.

[15] L. Krempasky, C. Schmidt. Theory of "supercurrents" and their influence on field quality and stability of superconducting magnets. J. Appl. Phys., 1995; 78 (9): 5800.

[16] N. Mitchell. Analysis of non-uniform current distribution effects in multistage cable-in-conduit conductors. Cryogenics, 1999; 39: 539.

[17] L. Bottura, M. Breschi, M. Fabbri. Analytical Solution for the Current Distribution in Multistrand Superconducting Cables. Paper submitted to J. Appl. Phys. to be published.

[18] L. Bottura, C. Rosso, M. Breschi. A General Model for Thermal, Hydraulic and Electric Analysis of Superconducting Cables. Cryogenics, 2000; 40: 617.

[19] M. Breschi, M. Fabbri. Analytical solutions for the current distribution in superconducting cables. DIEUB Internal Report. 2002; University of Bologna, Italy. 


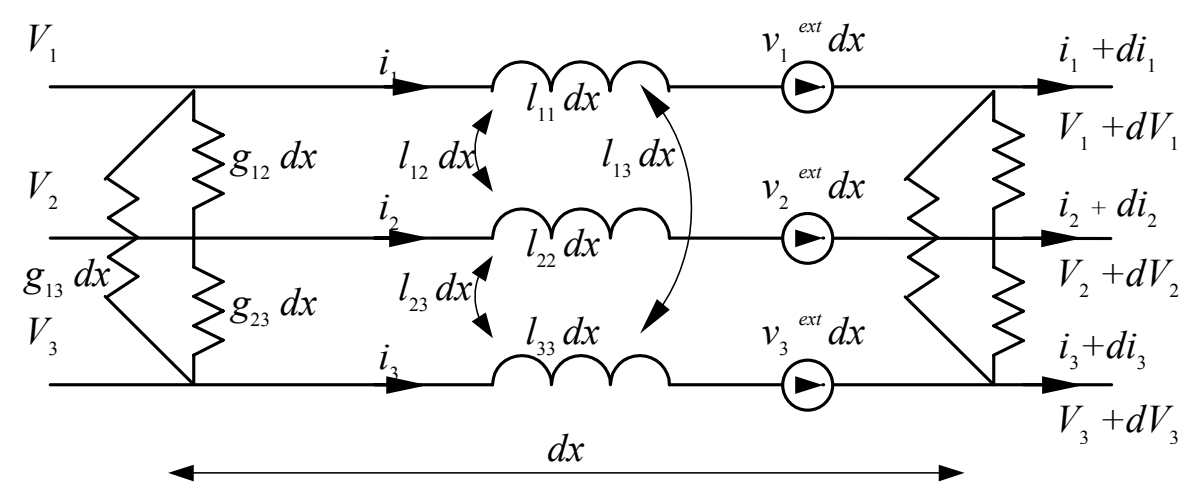

Fig. 1. Distributed parameters circuit model of the elemental mesh of cable used to describe current distribution in multistrand superconducting cables.

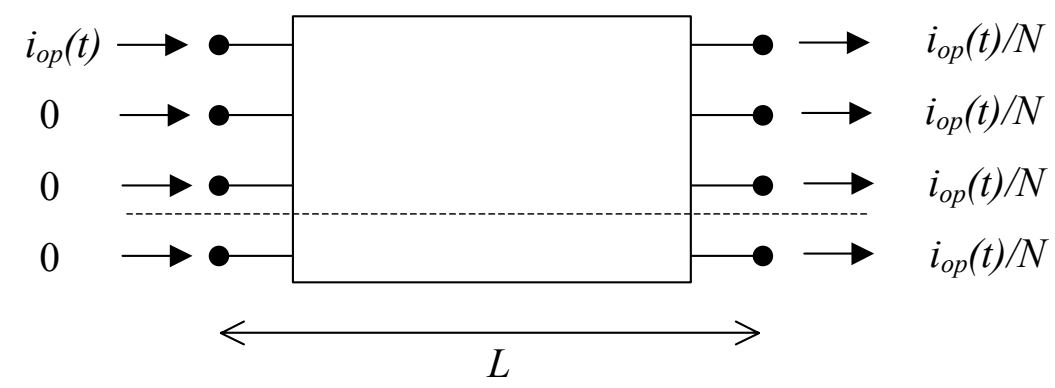

Fig. 2. Boundary conditions considered for the $N$-strand cable.
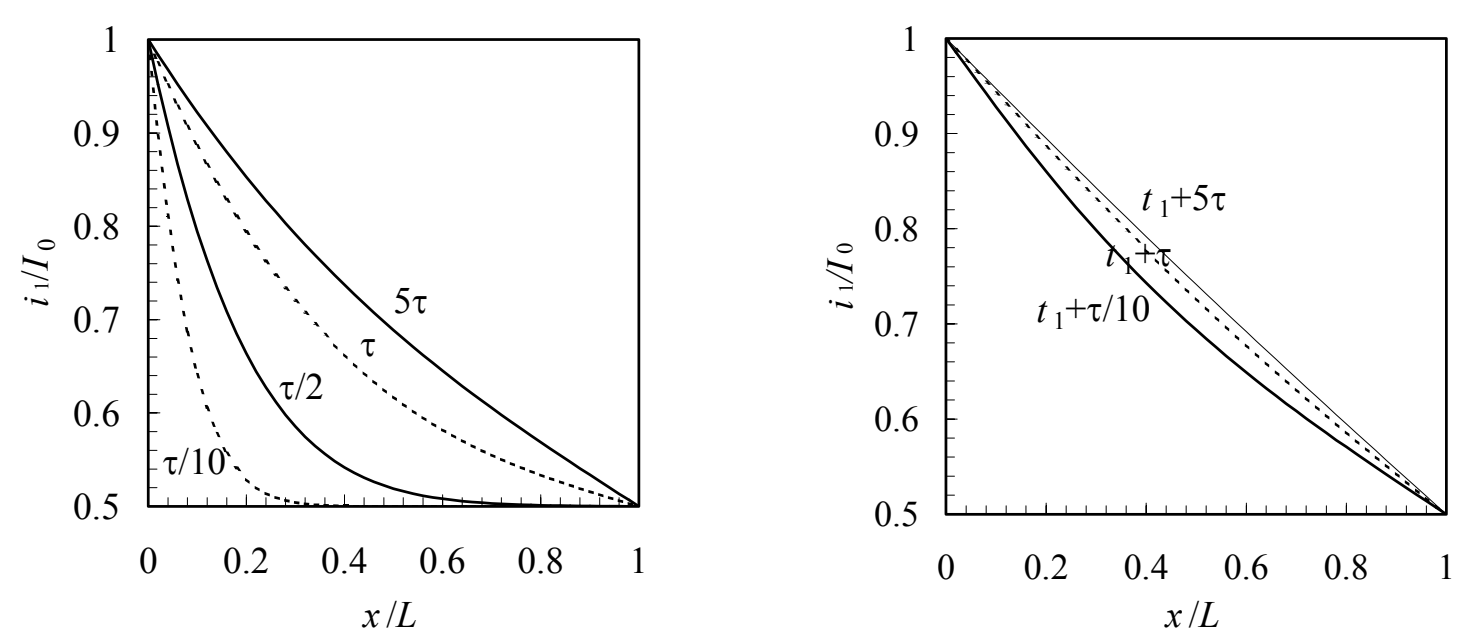

Fig. 3. Evolution of the current in the first strand of a two-strand cable with the parameters discussed in the text and subjected to non uniform boundary conditions. The current at each time step reported is normalised to the value $I_{0}$ of the current at $x=0$. The cable is subjected to a current ramp until $t_{1}$ equal to $5 \tau$, followed by a current plateau. The left plot reports the current rise during the current ramp for $t<t_{1}$, while the right plot shows the current plateau for $t>t_{1}$. 

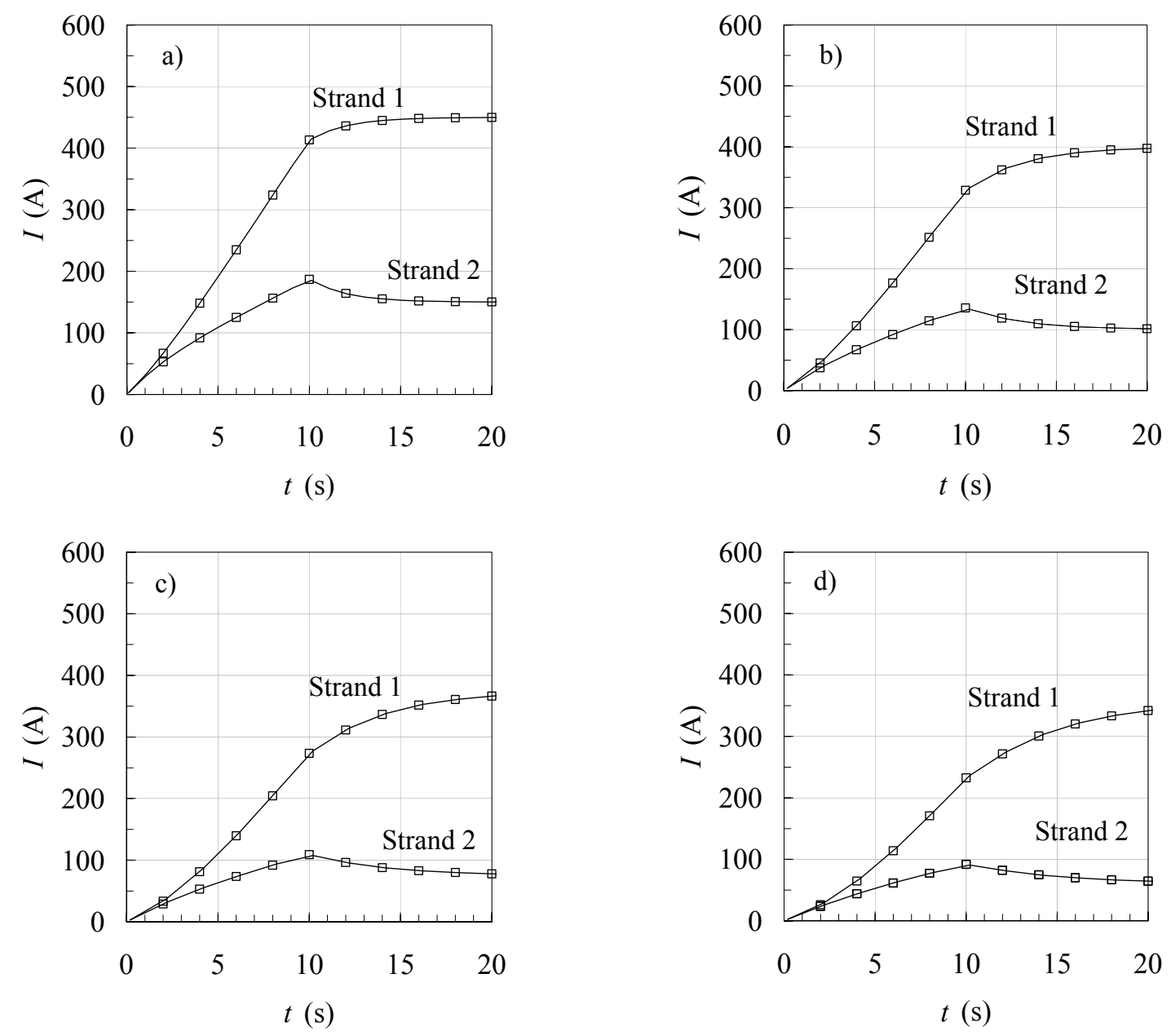

Fig. 4. Time evolution of the strand currents in the middle of the cable for cables made of 2, 3, 4, 5 strands (plots are marked respectively a), b), c), d)) with the boundary conditions specified in the text. All strands except strand \#1 have the same current as strand \#2. A comparison between the analytical solution (symbols), and the numerical simulation (lines) shows a good agreement.

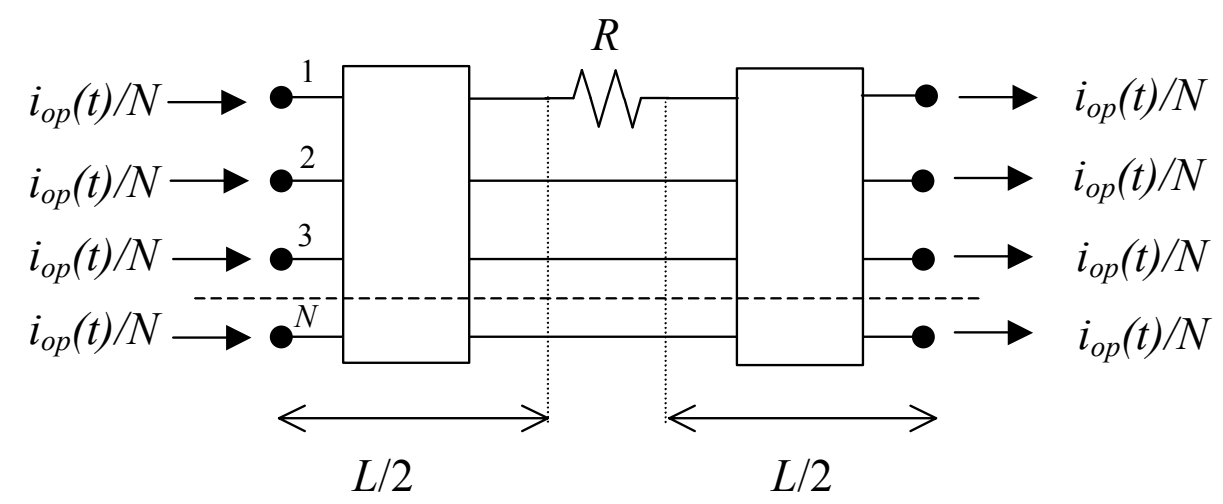

Fig. 5. Simplified model for quench in strand $\# 1$ in the middle of the cable. 


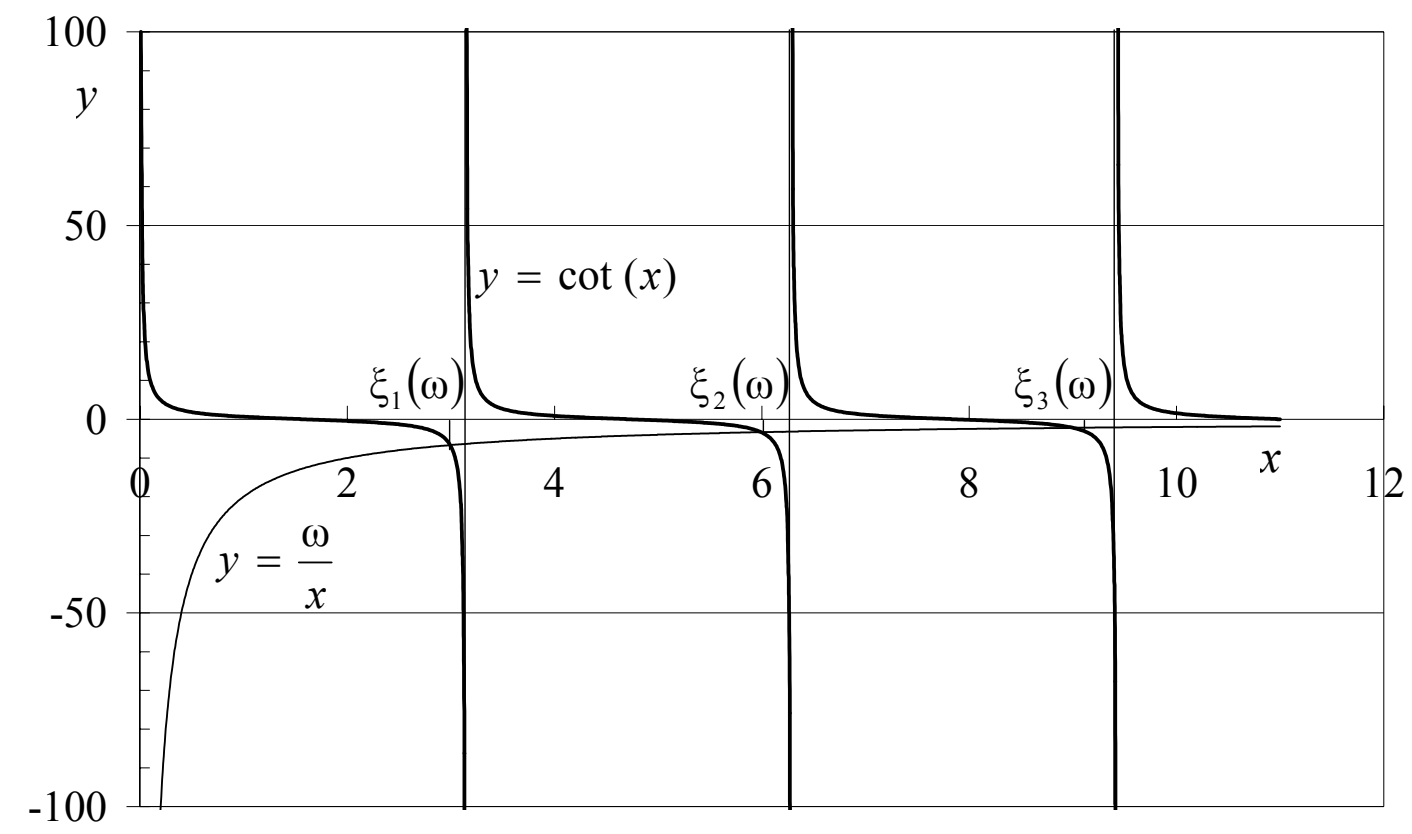

Fig. 6. Points of contact between the $\cot (x)$ and $\frac{\omega}{x}$ functions. The solutions are bounded as $\left(n-\frac{1}{2}\right) \pi<\xi_{n}(\omega)<n \pi$ and they approach $\left(n-\frac{1}{2}\right) \pi$ as $n$ increases.
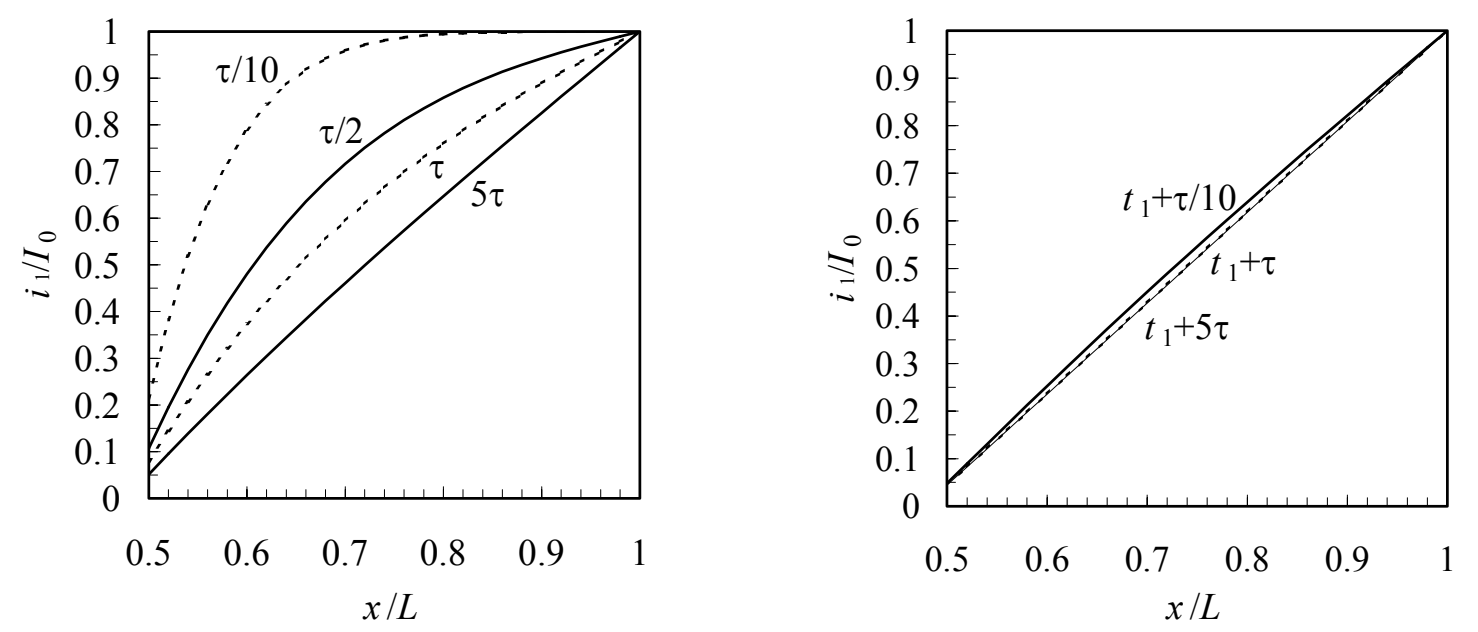

Fig. 7. Evolution of the current in the first strand of a two-strand cable with a lumped resistance in the middle of strand \#1 simulating quench. The current at each time step reported is normalised to the value $I_{0}$ of the current at $x=0$. The cable is subjected to a current ramp until $t_{1}$ equal to $5 \tau$, followed by a current plateau. The left plot reports the current rise during the current ramp for $t<t_{1}$, while the right plot shows the current plateau for $t>t_{1}$. 

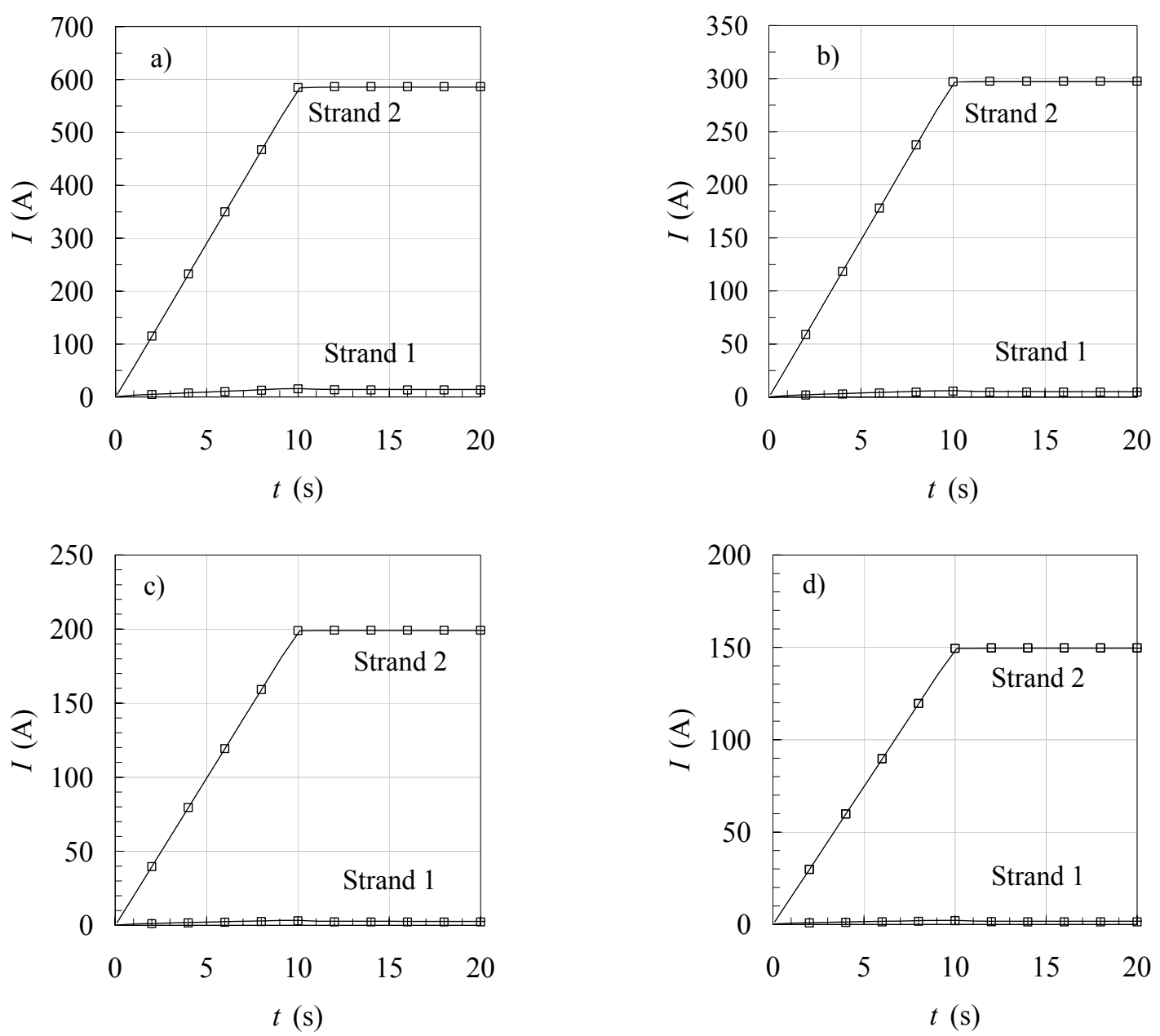

Fig. 8. Time evolution of the strand currents in the middle of the cable for cables made of 2, 3, 4, 5 strands (plots are marked respectively a), b), c), d)) with a lumped resistance in the middle of strand \#1 simulating quench. All strands except strand \#1 have the same current as strand \#2. A comparison between the analytical solution (symbols), and the numerical simulation (lines) shows a good agreement.
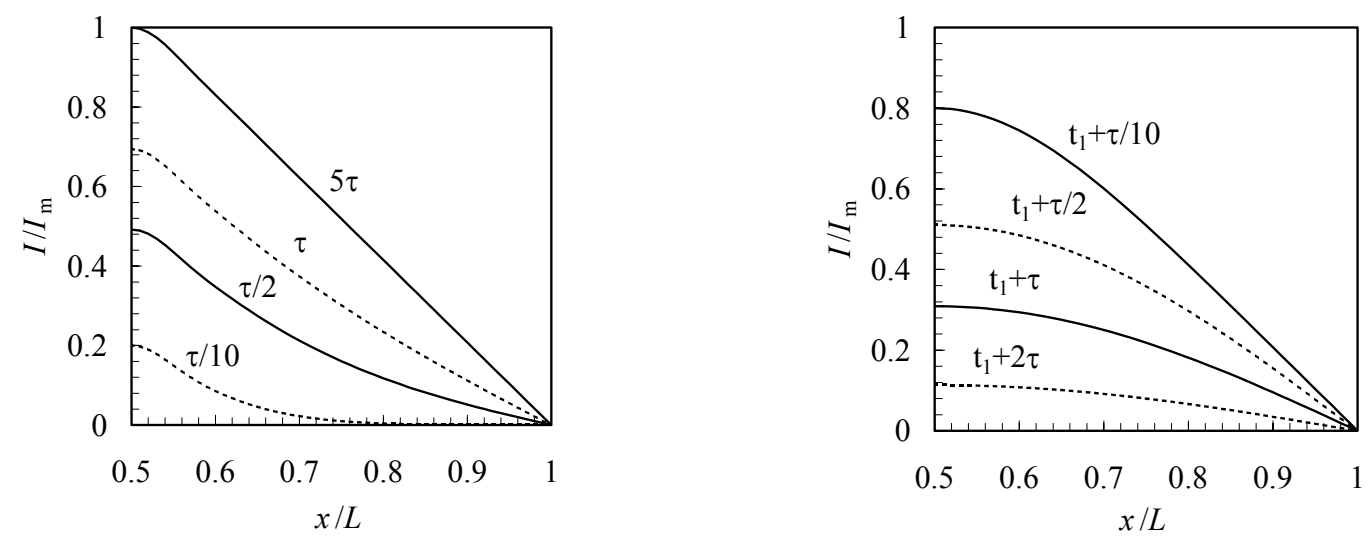

Fig. 9. Evolution of the current in the first strand of a two-strand cable with an external voltage acting in the middle of strand \#1. The current at each time step reported is normalised to the maximum value that could be reached after a time much longer than the time constant. The cable is subjected to this external voltage until $t_{1}$, while the excitation is nil after $t_{1}$. The left plot reports the current rise during the field ramp for $t<t_{1}$, while the right plot shows the current decay for $t>t_{1}$. 

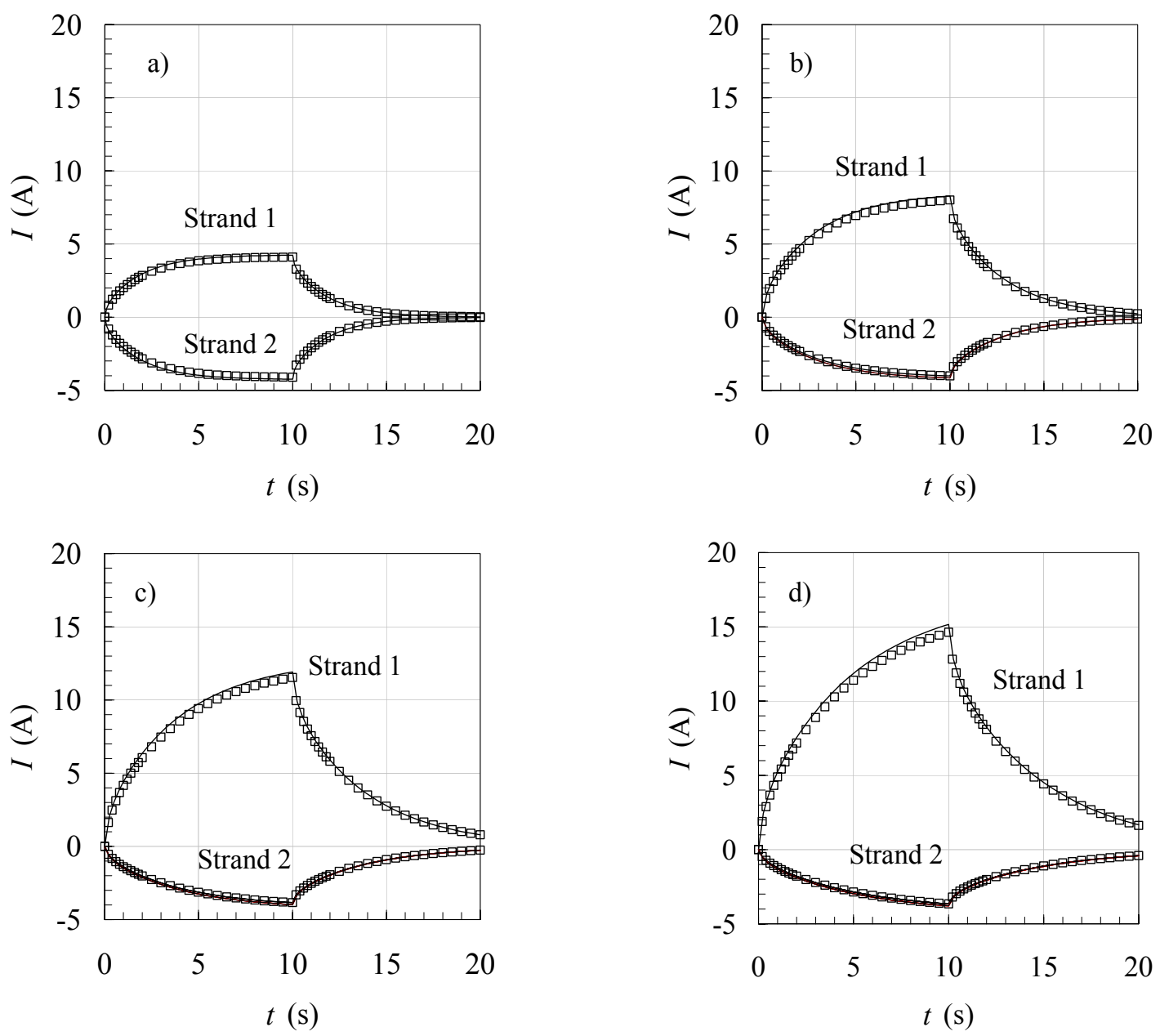

Fig. 10. Time evolution of the strand currents in the middle of the cable for cables made of 2, 3, 4, 5 strands (plots are marked respectively a), b), c), d)) with an external driving voltage acting on strand \#1 in the middle of the cable. All the strands except strand \#1 have the same current as strand \#2. A comparison between the analytical solution (symbols), and the numerical simulation (lines) shows a good agreement. 